\title{
New P-chirogenic diphosphines and their use in catalytic asymmetric reactions*
}

\author{
Tsuneo Imamoto \\ Department of Chemistry, Faculty of Science, Chiba University, Inage-ku, Chiba \\ 263-8522, Japan
}

Abstract: Enantiomerically pure $(S, S)$-1,2-bis[(o-alkylphenyl)phenylphosphino]ethanes $\left(o\right.$-alkylphenyl $=o$-methylphenyl, $o$-ethylphenyl, $o$-isopropylphenyl, $5^{\prime}, 6^{\prime}, 7^{\prime}, 8^{\prime}$-tetrahydronaphthyl), (S,S)-1,2-bis(alkyl-methylphosphino)ethanes (alkyl = $t$-butyl, 1-adamantyl, 1-methylcyclo-hexyl, 1,1-diethylpropyl, cyclopentyl, cyclohexyl) (abbreviated as BisP*), and $(R, R)$-1,1-bis(alkylmethylphosphino)methanes (alkyl = isopropyl, $t$-butyl, cyclohexyl, phenyl) (abbreviated as MiniPHOS) were prepared from phosphorus trichloride via short routes using phosphine-boranes as intermediates. The crystal structures of cationic rhodium complexes, $\left[\mathrm{Rh}((S, S)-1,2\right.$-bis((phenyl)(5',6',7',8'-tetrahydronaphthyl)phosphino)ethane)(cod) $] \mathrm{BF}_{4}$, $[\mathrm{Rh}((S, S)-t-\mathrm{Bu}-\mathrm{BisP} *(\mathrm{nbd}))] \mathrm{BF}_{4}$, and $\left[\mathrm{Rh}((R, R)-t \text {-Bu-MiniPHOS })_{2}\right] \mathrm{PF}_{6}$, clearly indicate the ideal asymmetric environments. These ligands exhibited an excellent to almost perfect level of enantioselectivity in asymmetric hydrogenations of $\alpha$-dehydroamino acid derivatives and other catalytic asymmetric reactions.

\section{INTRODUCTION}

Optically active diphosphines play an important role as chiral ligands in various metal-catalyzed asymmetric reactions, and numerous chiral diphosphines have been designed and synthesized over the past three decades [1,2]. Nevertheless, the development of more efficient ligands is still required, and we have intended to explore new useful chiral diphosphines. Among various chiral phosphines, our attention has been focused on P-chirogenic phosphine ligands like DIPAMP [3], because this class of phosphines possesses chirality at phosphorus, and hence the imposed asymmetric environment leads to high enantioselectivity. Here we report the design and the synthesis of new P-chirogenic diphosphines and their use in representative metal-catalyzed asymmetric reactions.

\section{OPTICALLY PURE 1,2-BIS[(o-ALKYLPHENYL)PHENYLPHOSPHINO]ETHANES}

Previously, we studied synthesis and reactions of phosphine-boranes and found that optically active ethano-bridged diphosphines possessing chiral centers at the phosphorus atoms can be synthesized from chiral phosphine-boranes having methyl group [4]. Scheme 1 shows the outline of our methodology.

In this reaction sequence, the boranato group plays two roles: the protection of air-sensitive phosphines and the activation of the adjacent methyl group. It is also noted that optical amplification is

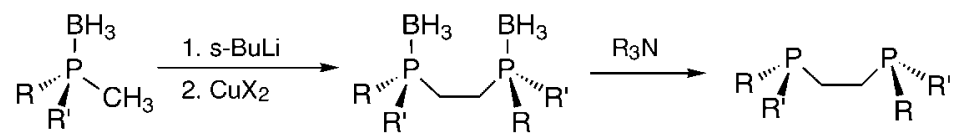

Scheme 1 Synthesis of P-chirogenic bis(phosphino)ethanes.

*Lecture presented at the XIX ${ }^{\text {th }}$ International Conference on Organometallic Chemistry (XIX ICOMC), Shanghai, China, 23-28 July 2000. Other presentations are published in this issue, pp. 205-376. 


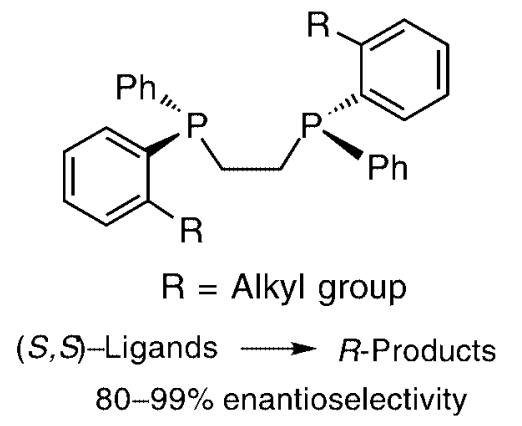

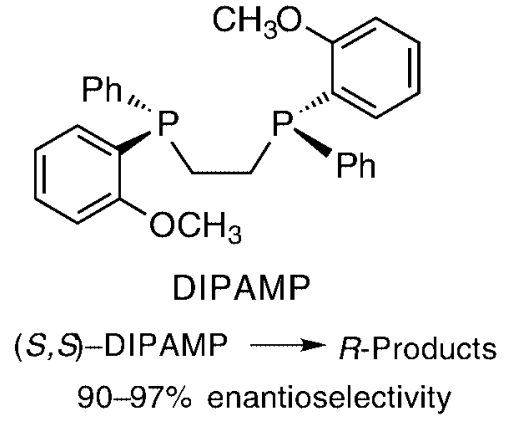

Fig. 1

observed at the dimerization step; for example, diphosphine-boranes with $>99 \%$ ee after removal of the meso isomer can be obtained from the starting phosphine-boranes with $90 \%$ ee.

Based on this methodology, enantiomerically pure $(S, S)$-1,2-bis[(o-alkylphenyl)phenylphosphino]ethanes $(o$-alkylphenyl $=o$-methylphenyl, $o$-ethylphenyl, $o$-isopropylphenyl, 5',6',7',8'-tetrahydronaphthyl) were prepared from dichlorophenylphosphine in four steps. The obtained ligands were converted to the corresponding rhodium complexes, which were tested for asymmetric hydrogenation of dehydroamino acid derivatives. The reactions were carried out under the same conditions as the ones employed by Knowles et al. in order to compare their results with ours.

The newly synthesized diphosphines having ortho-alkyl group provided high to excellent asymmetric induction (80->99\%). The sense of stereoselection is the same as in the case of hydrogenation with DIPAMP-Rh (Fig. 1). These results clearly indicate that the ortho-alkyl group in these ligands is as good as the methoxy group in DIPAMP. Thus, the steric bulkiness of the substituents on phosphorus is the most important factor affecting the stereoselection, rather than the coordinative interaction of ortho-methoxy group [5].

\section{P-CHIROGENIC 1,2-BIS(ALKYLMETHYLPHOSPHINO)ETHANES (BisP*) AND 1,1-BIS(ALKYLMETHYLPHOSPHINO)METHANES (MiniPHOS)}

The studies mentioned above led us to explore more efficient and practically useful P-chirogenic phosphine ligands. Newly designed phosphine ligands are 1,2-bis(alkylmethylphosphino)ethanes (alkyl = tert-butyl, 1,1-diethylpropyl, 1-adamantyl, 1-methylcyclohexyl, cyclopentyl, cyclohexyl) (abbreviated as BisP*). An important feature of these ligands is that a bulky alkyl group and the smallest alkyl group (methyl group) are bonded to each phosphorus atom. These ligands form five-membered $C_{2}$-symmetric chelates, and the imposed asymmetric environment leads to high enantioselectivity in asymmetric reactions. These trialkylphosphine ligands are electron-rich and provide very high catalytic efficiencies in transition metal-catalyzed homogeneous hydrogenations.

The chiral diphosphines (BisP*) and their rhodium complexes were synthesized according to Scheme 2 . It is noted that the desired optically pure phosphine ligands were obtained in only three steps from trichlorophosphine.

The rhodium complexes were employed in asymmetric hydrogenation of dehydroamino acid derivatives. The representative hydrogenation results are illustrated in Fig. 2. Very high enantioselection exceeding $99 \%$ was observed when the ligands having tert-butyl, 1-adamantyl, or 1-methylcyclohexyl group at the phosphorus were employed [6-8]. These results indicate that changing the bulky alkyl group controls the enantioselection. Among these ligands, tert-butyl BisP* is the most useful, because this ligand is synthesized easily using inexpensive materials and it provides very high enantioselection in the reaction of various $\alpha$-aminoacrylates, itaconic acid derivatives, and related substrates. 


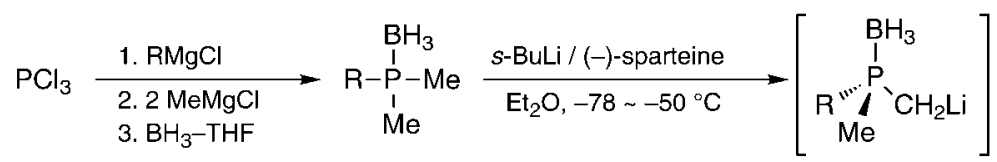

$$
\begin{aligned}
& \longrightarrow \mathrm{CuCl}_{2}
\end{aligned}
$$

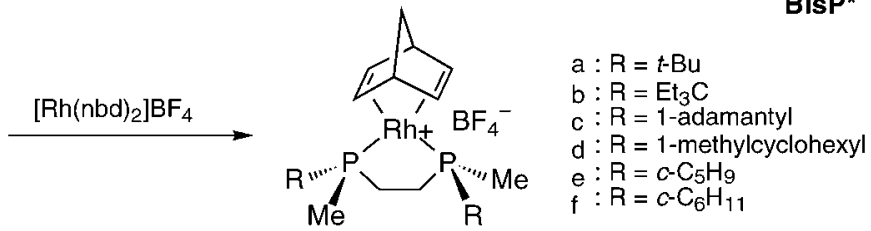

Scheme 2 Synthesis of BisP* from trichlorophosphine.<smiles>CP(CCP[P](C)(C)C)C(C)(C)C</smiles>

$>99 \%$

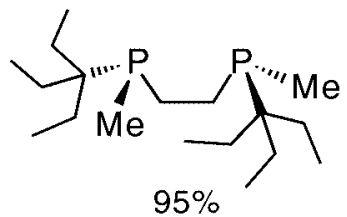

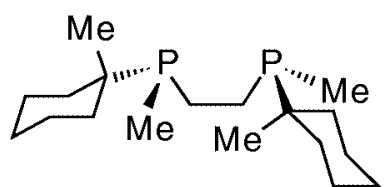

$>99 \%$

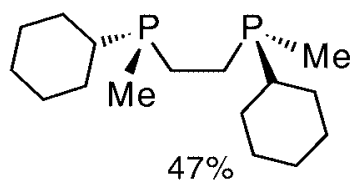

$47 \%$
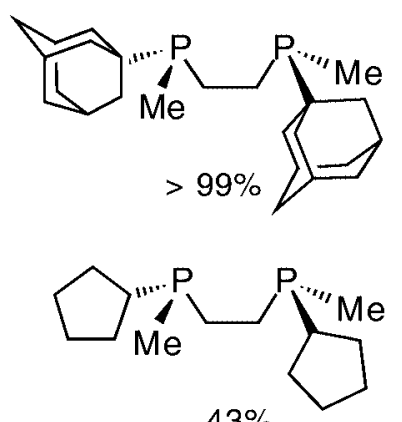

$43 \%$

Fig. 2 Enantioselectivity in the rhodium-catalyzed hydrogenation of methyl $(Z)$ - $\alpha$-acetylaminocinnamate.

We next challenged the design of the effective ligands of minimal possible size. The designed ligands are 1,1-bis(alkylmethylphosphino)methane (abbreviated as MiniPHOS). These ligands were prepared by a procedure similar to that applied for the synthesis of Bis $\mathrm{P}^{*}$ and converted to the corresponding rhodium complexes (Scheme 3).

The rhodium complexes were used as catalyst precursors in asymmetric hydrogenation of various dehydroamino acids and their methyl esters. Almost complete enantioselectivity was achieved for the hydrogenation of several $\alpha$-acetamidocinnamic acid derivatives, when the ligands having tert-butyl, cyclohexyl, and isopropyl groups were used. Moreover, these catalysts were also found to be effective for the $\beta, \beta$-disubstituted enamides which are normally difficult substrates for asymmetric hydrogenations $[8,9]$.

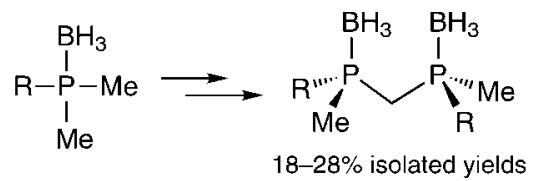

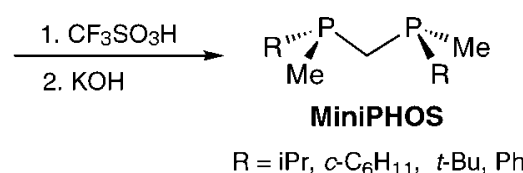

Scheme 3 Synthesis of MiniPHOS. 
BisP* and MiniPHOS were employed as the chiral ligands in other catalytic asymmetric reactions. The results summarized in equations [9-12] clearly indicate that these ligands are potentially useful for various transition metal-catalyzed asymmetric reactions.

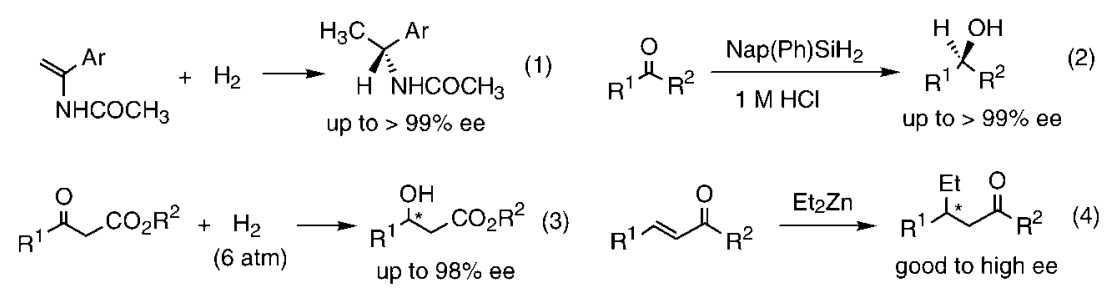

\section{CONCLUSION}

New P-chirogenic ethano- or methylene-bridged diphosphines were designed and synthesized via phosphine-boranes as intermediates. Among these, BisP* and MiniPHOS are extremely useful as the chiral ligands in representative catalytic asymmetric reactions such as rhodium-catalyzed hydrogenations, although they are quite simple and small in comparison with the previously reported chiral ligands.

\section{ACKNOWLEDGMENTS}

The author thanks his coworkers, whose names are listed in the references. This work was supported by the Research for the Future program, the Japan Society for the Promotion of Science.

\section{REFERENCES}

1. R. Noyori. Asymmetric Catalysis in Organic Synthesis, Wiley, New York (1994).

2. I. Ojima (Ed.). Catalytic Asymmetric Synthesis, VCH, New York (1993).

3. W. S. Knowles, M. J. Sabacky, B. D. Vineyard, D. J. Weinkauff. J. Am. Chem. Soc. 97, 2567 (1975).

4. T. Imamoto, T. Kusumoto, N. Suzuki, K. Sato. J. Am. Chem. Soc. 107, 5301 (1985).

5. T. Imamoto, H. Tsuruta, Y. Wada, H. Masuda, K. Yamaguchi. Tetrahedron Lett. 36, 8721 (1995).

6. T. Imamoto, J. Watanabe, Y. Wada, H. Masuda, H. Yamada, H. Tsuruta, S. Matsukawa, K. Yamaguchi. J. Am. Chem. Soc. 120, 1635 (1998).

7. I. D. Gridnev, N. Higashi, K. Asakura, T. Imamoto. J. Am. Chem. Soc. 122, 7183 (2000).

8. I. D. Gridnev, Y. Yamanoi, N. Higashim, H. Tsuruta, M. Yasutake, T. Imamoto. Adv. Synth. Cat. 343, 118 (2001).

9. Y. Yamanoi and T. Imamoto. J. Org. Chem. 64, 2988 (2000).

10. I. D. Gridnev, N. Higashi, T. Imamoto. J. Am. Chem. Soc. 122, 10486 (2000).

11. I. D. Gridnev and T. Imamoto. Organometallics 20, 545 (2000).

12. T. Yamano, N. Taya, M. Kawata, T. Huang, T. Imamoto. Tetrahedron Lett. 40, 2577 (1999). 